\title{
Miniaturized Multi-spurline Bandstop Filter Design with a Meandered Slot Lines
}

\author{
Bhanu Shrestha $^{1}$, Surendra Shrestha ${ }^{2}$ \\ ${ }^{1}$ Department of Electronics Engineering, Kwangwoon University, Seoul, Korea \\ Email:bnu@kw.ac.kr \\ ${ }^{2}$ Department of Electronics \& Computer Engineering, Pulchowk Campus, \\ Institute of Engineering, Tribhuvan University, Nepal \\ corresponding author: surendra@ioe.edu.np
}

\begin{abstract}
A multi-spurlines with meandered slot line bandstop filter is studied. A wideband width bandstop filter is designed and simulated on the basis of a Teflon substrate having thickness of $0.54 \mathrm{~mm}$ and a dielectric constant of 2.54 . The wideband width is achieved even if the multi-coupling effects of multi-spurline are increased. The proposed bandstop filter has good S-parameter responses with good stopband characteristics. The insertion loss (S21) and return losses are $34.5 \mathrm{~dB}$ and $0.12 \mathrm{~dB}$ respectively. The wide band rejection depth increased by improving transmission zeros at $12 \mathrm{GHz}$. The wideband bandstop filter can be implemented to the $\mathrm{X}$-band applications. The size of the designed bandstop filter is $5.8 \mathrm{~mm} \times 3 \mathrm{~mm}$.
\end{abstract}

Key words : Multi-spurline, microwave bandstop filter, spurlineBSF, meandered slot lineBSF.

\section{Introduction}

Wireless communication systems require a wide bandstop filter to reject the unnecessary frequencies in order to have desired frequency. So, a high performance bandstop filter is necessary to mitigate the problem of extracting the desired signal from the transceiver systems [1]. The $Q$ factor of the resonator plays an important role in the system to achieve a narrow band and to reject the unwanted frequencies, however, in this design; the bandstop filter is built with wideband for the same purpose. The trend of using different types of bandstop filters such as stepped impedance resonators (SIR) bandstop filter, split ring resonators (SRR) filter, bandstop filter with spiral resonators, hairpin resonators, spurline resonator filters, meandered slot line resonator bandstop filters, wideband bandstop filters and so on. These filters can be used for the transmitter and receiver of the wireless communication systems [2-7]. Therefore, many researchers are making an effort to enhance the performance of bandstop filters with different patterns with stable S-parameter responses. We also require a low cost, low power and a simple design with significantly good circuit performance for the system. A multi-spurline with meandered slot line structure is used, which is a good candidate for the bandstop resonator fitler design, because they have good coupling effect and high $Q$ at the same time. Moreover, this simple structure is miniaturized, which gives bandstop characteristics and stability with temperature to some extent. In addition, when we add the multi-spurline with meandered slot line structure, the frequency responses can be improved and 
the feasibility of fabricating microstrip multi-spurlines with meandered slot lines bandstop filter is verified. Such works can also be feasibly integrated into MMICs with various technologies such as $\mathrm{InGaP} / \mathrm{GaAs} \mathrm{HBT}$ and so on [8].

\section{Design and Simulations}

The multi-spurline with a meandered slot line resonator was designed and simulated to achieve high performance bandstop filter with good frequency S-parameter responses. The bandstop filter is simulated using the SONNET Lite 3D planar EM simulator.

The miniaturized multi-spurline with a meandered slot line bandstop filter is designed and simulated which is based on a Teflon substrate with a thickness and a dielectric constant of 0.54 $\mathrm{mm}$ and 2.54, respectively. The schematic of the multi-spurline with meandered slot line bandstop filter is depicted in Figure 1. The structure of the multi-spurline has a $0.15 \mathrm{~mm}$ slot width, and the slot height ranges from $0.35 \mathrm{~mm}$ to $1.35 \mathrm{~mm}$ in the upper and lower parts, which are diagonally symmetric. In the same manner, the slot line is meandered with a diagonal symmetry as well, and its width is also $0.15 \mathrm{~mm}$. The meandered slot line has a length of $7.5 \mathrm{~mm}$ in diagonal symmetry with a central line of $2.25 \mathrm{~mm}$. The slot gap of the multi-spurline provides a capacitive effect while the narrow line exhibits an inductive effect. The meandered slot line provides an inductor and resistor effect [8]. Due to the effective permittivity of the dielectric substrate, the effective inductance and capacitance of the microstrip line are improved therefore, it has potential to achieve a low phase noise if we implement in a microwave oscillator. The spurlines with a meandered slot line are easy to fabricate because they require only single-sided board etching. The Teflon substrate gives rigidity and concentrates the field density between the slot gaps, suppressing higher-order modes or radiation. The loaded $Q$ can be found using the $3 \mathrm{~dB}$ bandwidth of the insertion loss, which is given by $Q_{\mathrm{L}}=\omega_{0} / \Delta \omega$. The proposed bandstop filter can be etched on a $50 \Omega$ microstrip line $1.5 \mathrm{~mm}$ wide with a reference line of $5.55 \mathrm{~mm}$. The specification for the resonant frequency is set to $9.2 \mathrm{GHz}$ before designing it.

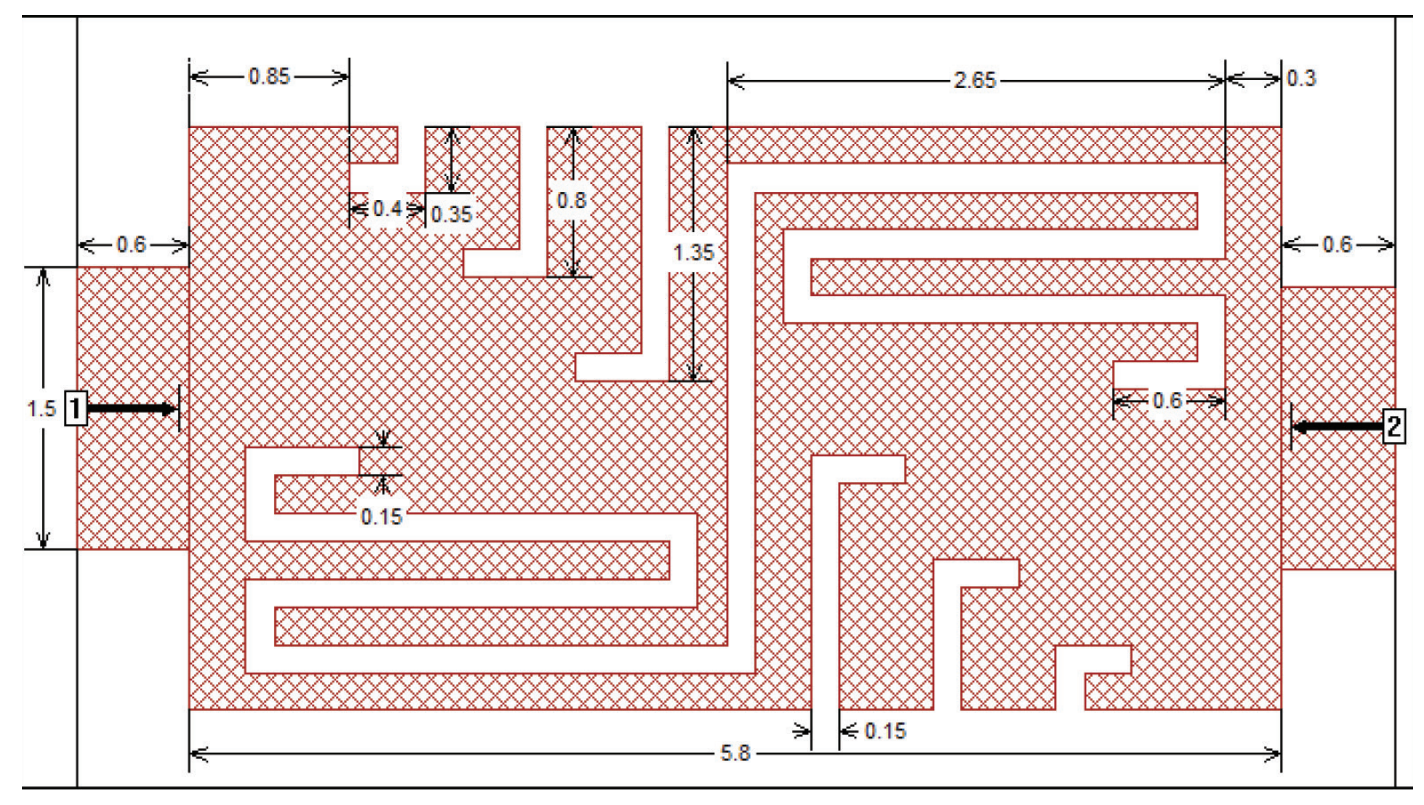

Figure 1. Schematic design layout of the proposed resonator (unit: $\mathrm{mm}$ ) 


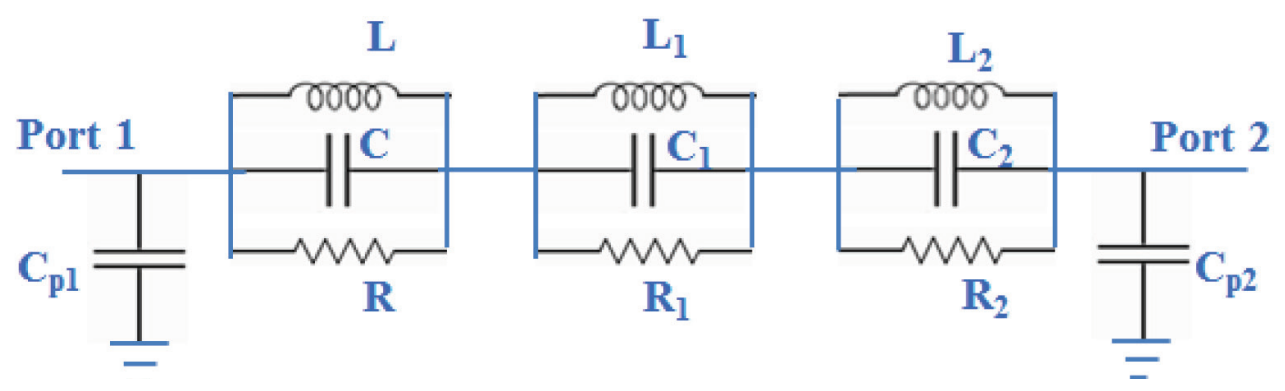

Figure 2. Equivalent circuit of the proposed resonator

In the equivalent circuit shown in Figure 2, the $C_{\mathrm{p} 1}$ and $C_{\mathrm{p} 2}$ are the parasitic capacitance effects in port 1 and 2 respectively. The RLC networks are parallel arranged accordingly as the multispurlines in the design pattern, The RLC components in the equivalent circuits can be calculated using equations (1), (2) and (3), and we can also extract the component values from the simulation [9-10].

$$
\begin{gathered}
R=\left.2 Z_{0}\left(\frac{1}{\left|S_{21}\right|-1}\right)\right|_{f=f_{0}} \\
C=\frac{2 \omega_{c}}{2 Z_{0}\left(\omega^{2}{ }_{0}-\omega_{c}{ }^{2}\right)} \\
L=\frac{1}{\left(4 \pi f_{0}\right)^{2} C}=\frac{1}{2 \omega^{2} C}
\end{gathered}
$$

where $S_{21}$ is the transmission co-efficient, $Z_{\mathrm{o}}$ is the characteristics impedance of $50 \Omega, \omega$ is the angular frequency and $\omega_{\mathrm{c}}$ is the $3 \mathrm{~dB}$ cut-off angular frequency (where $\omega=2 \pi f$ ).

The simulation results of the multi-spurline resonator filter is depicted in Figure 3 which shows S-parameter responses. The simulated bandstop filter exhibited $0.12 \mathrm{~dB}$ as its insertion loss (S21) and 34.5 as its return loss (S11). Both parameters are very important for the system. The higher the value of return loss, the higher the band rejection depth and that can suppress the harmonics as well as spurious frequencies in the system. The wider band rejection is essential for the system too. So, we designed wideband bandstop filter in this work. In this design, we have achieved 2 $\mathrm{GHz}$ bandwidth in simulation result. In the passband, the transmission zero is also very good which shows over $-40 \mathrm{~dB}$. The overall size of the designed bandstop filter is $5.8 \mathrm{~mm}$ x $3 \mathrm{~mm}$ which very compact and it also can be possible to fabricate in microwave monolithic integrated circuit (MMIC) technology. The performance of the BSF is summarized in Table 1. 


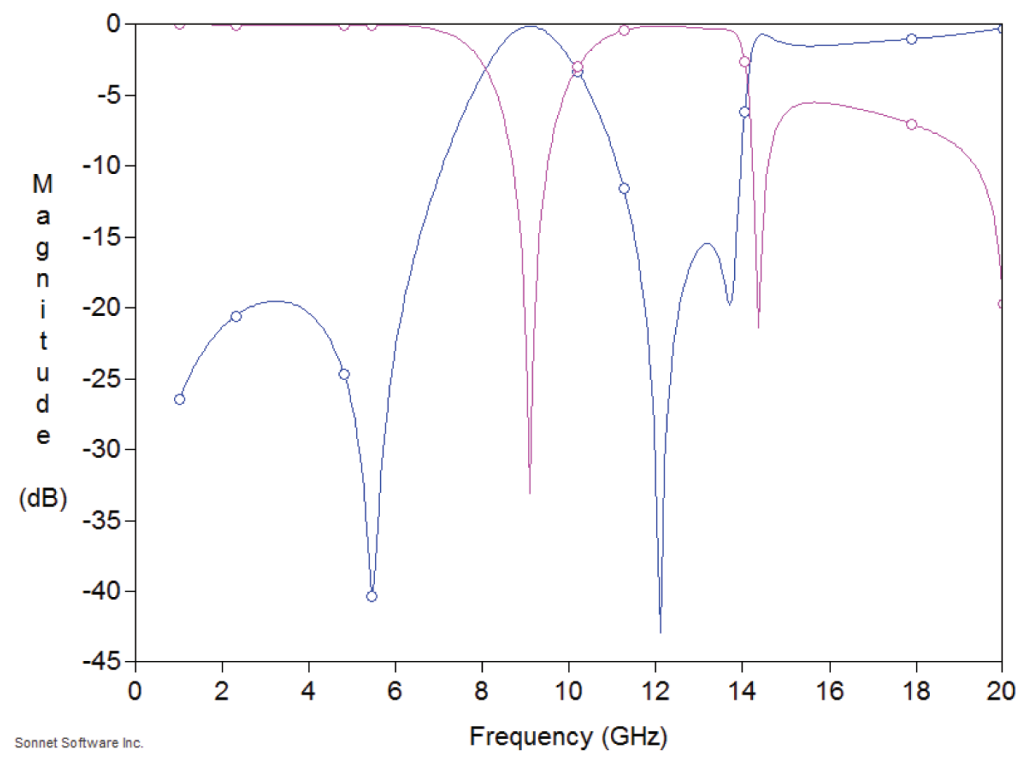

Figure 3. S-parameter response of the simulated bandstop filter.

Table 1: Performance of the BSF

\begin{tabular}{|c|c|c|}
\hline Parameters & Units & Simulation Results \\
\hline Operating Frequency & $\mathrm{GHz}$ & 9.2 \\
\hline Insertion Loss (S21) & $\mathrm{dB}$ & 34.5 \\
\hline Return Loss (S11) & $\mathrm{dB}$ & 0.12 \\
\hline Bandwidth & $\mathrm{GHz}$ & 2 \\
\hline Size & $5.8 \times 2.8$ & \\
\hline
\end{tabular}

\section{Conclusion}

The mini aturized multi-spurline with meandered slot line bandstop filter is designed and simulated on the based on Teflon substrate with a dielectric constant of 2.54. The purposed miniaturized bandstop filter has flexibility in terms of coupling effects. The designed bandstop filter has the return loss over 0.12 and insertion loss over $30 \mathrm{~dB}$ which play a great role in the filter performance. The design is made a higher electromagnetic coupling effect in order to enhance the performance. The designed BSF can be implemented to X-band applications in microwave communication systems. The size is very compact due to use of meander slot line and such pattern has the potential for MMIC application to make smaller and smaller in size too.

Acknowledgment: This work was also supported by a Research Grant from Kwangwoon University, South Korea in 2015. 


\section{References}

[1] Rohde UL, Poddar AK, and Bock G (2005), The Design of Modern Microwave Oscillators, Theory and Optimization, Wily, New York, NY.

[2] Maharjan RK, Shrestha B and Kim NY(2012), Microstrip Cross-coupled Interdigital SIR Based Bandpass Filter, Radio Engineering, 211210-2512.

[3] Jung JS et al. (2006), Power Enhancement of Microwave Oscillator Using a High-Q SpiralShaped DGS Resonator, Conf. Proceedings.

[4] Shrestha B and Kim NY (2012), Microwave Oscillator Design Using Folded T-type Slot Resonator for X-band Applications, Microwave and Optical Technology Letters (MOTL), 54 2817-2821.

[5] Liu H, Knoechel RH and Schuenemann KF (2007), Miniaturized Bandstop Filter Using Meander Spurline and Capacitively Loaded Stub, Electronics and Communications Research Institute (ETRI) Journal 29 614-618.

[6] Tu WH and Chang K (2005), Compact MicrostripBandstop Filter Using Open Stub and Spurline, IEEE Microw.and Wireless Compo. Lett., 15, 268-270

[7] Shrestha B and Kim NY (2015), Microstrip wideband bandstop filter with open stubs for UWB applications, Microwave and Optical Technology Letters (MOTL), 57 (4), 1003-1006.

[8] Shrestha B and Kim NY (2010), Design of Low Phase Noise InGaP/GaAs HBT Based Differential Colpitts VCOs for Interference Cancellation System, Indian Journal of Engineering \& Materials Sciences (IJEMS) 17 34-38.

[9] Liu H, Sun L and Shi Z (2007), Dual Bandgap Characteristics of Spurline Filters and its Circuit Modeling, Microwave and Optical Technology Letters (MOTL) 49, 2805-2807.

[10] Shrestha B and Kim NY(2015), Adjustable Wideband BandstopFilter with Inductive Coupling for Ultrawideband applications, Microwave and Optical Technology Letters (MOTL), 57(8) 1901-1905. 